\section{Respeto a los derechos de las personas que se atienden en Servicios de Psiquiatría Ambulatoria en Chile}

\author{
ALBERTO MINOLETTI ${ }^{1}$, OLGA TORO ${ }^{2, a}$, RUBÉN ALVARADO ${ }^{1}$, \\ CLAUDIA CARNIGLIA $^{3, \mathrm{~b}}$, ALEJANDRO GUAJARDO ${ }^{4, \mathrm{~b}}$, XIMENA RAYO $^{1, \mathrm{c}}$
}

\section{A survey about quality of care and user's rights in Chilean psychiatric services}

\begin{abstract}
Background: Quality of care and respect for the rights of users are critical to achieve positive health outcomes and respond appropriately to the expectations of people, particularly if they have mental illnesses. Aim: To carry out a baseline diagnosis of quality of care and respect for rights in public outpatient psychiatric services. Material and Methods: Quality of care and respect for patients' rights was assessed by a mental health professional and a trained psychiatric service user in 15 ambulatory psychiatric services. The WHO QualityRights instrument was used, reviewing documentation and making observations in each facility, as well as interviewing 146 patients, 148 health care workers and 64 relatives of patients. Results: A high level of achievement was accomplished in terms of discrimination-free health care, availability of psychotropic medications, lack of abuse or neglect and use of informed consents. A low level of achievement was found in terms of user support to cope with community living, access to education or work and participation in community activities, respect for user treatment preferences and preventive measures to avoid maltreatment and cruelty. Conclusions: Chile could improve the performance of psychiatry services having laws based on the "Convention of Rights of Persons with Disabilities" and standards of the World Health Organization, having national policies about quality of care and rights of users, reinforcing the community work of mental health care teams, reinforcing and informing users about their rights and promoting research on interventions to improve the respect of their rights.
\end{abstract}

(Rev Med Chile 2015; 143: 1585-1592)

Key words: Ambulatory care; Health Services for persons with disabilities; Human rights.
'Unidad de Salud Mental, Escuela de Salud Pública Dr. Salvador Allende, Universidad de Chile 2Departamento de Estudios, Estadísticas y Control de Gestión, Hospital Santiago Oriente Dr. Luis Tisné.

${ }^{3}$ Unidad de Salud Mental, Servicio de Salud Metropolitano Sur.

${ }^{4}$ Escuela de Terapia Ocupacional, Universidad Andrés Bello.

apsicóloga.

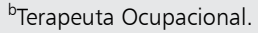

'Trabajadora Social.

Este trabajo contó con financiamiento del FONIS, proyecto SA1212073

Recibido el 31 de diciembre de 2014, aceptado el 7 de octubre de 2015.

Correspondencia a:

Dr. Alberto Minoletti

Av. Independencia 939, Santiago, Chile.

Teléfono: 056-2- 29786805 aminoletti2@gmail.com

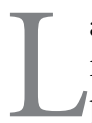

a calidad de atención y el respeto a los derechos de los usuarios son aspectos críticos para lograr resultados favorables en el tratamiento, evitar efectos indeseables y responder a las expectativas de los usuarios, particularmente en personas con enfermedades mentales ${ }^{1-5}$. La implementación del Plan Nacional de Salud Mental 2000 y la inclusión de cuatro problemas de salud mental en las garantías explícitas han permitido disponer en Chile de una cantidad mayor de re- cursos para salud mental en el sector público, logrando que un número creciente de personas sean atendidas $^{6-9}$. No obstante los avances logrados en acceso, algunos estudios han mostrado brechas de calidad de atención ${ }^{10}$ y no se encontraron estudios sobre el respeto de los derechos de los usuarios.

Una de las estrategias fundamentales para mejorar calidad en salud es la medición de indicadores del cumplimiento de estándares en los centros de atención ${ }^{1-5,11-14}$, la cual se asume puede 
producir efectos positivos a través de: motivar intrínsecamente a los equipos profesionales a mejorar aquellas prácticas con potencial para mejorar, motivar extrínsecamente a los equipos a mantener una buena reputación al comparar sus niveles de calidad con otros, e incentivar económicamente a los proveedores de modo de atraer un mayor número de usuarios.

En los últimos años ha existido una preocupación creciente en muchos países por los derechos humanos de las personas con enfermedades mentales, tanto en ámbitos civiles como en la utilización de servicios de salud ${ }^{15-16}$. Los derechos de estas personas se encuentran consignados en leyes nacionales y tratados internacionales, y en especial en la Convención de las Naciones Unidas sobre los Derechos de las Personas con Discapacidad, de la cual nuestro país es signatario desde el año $2008^{17}$.

Consecuentemente con el enfoque de mejora de calidad y con los fundamentos de derechos humanos, la Organización Mundial de la Salud (OMS) ha elaborado estándares y una metodología para medirlos ${ }^{18}$, los cuales pueden ayudar a los países a realizar avances en estas materias ${ }^{16,19-21}$. De acuerdo con los estándares de la OMS, el presente estudio se propuso efectuar un diagnóstico basal de la situación de calidad de atención y respeto de derechos en servicios de Psiquiatría Ambulatoria en el sistema público chileno. De esta manera, se pretende contribuir al desarrollo de estos servicios $y$, eventualmente, al bienestar y calidad de vida de las personas con enfermedades mentales.

\section{Material y Método}

Se efectuó un estudio transversal, teniendo como unidad de análisis los centros especializados de atención ambulatoria en salud mental, pertenecientes a las redes de atención pública y que atienden personas con primer episodio de esquizofrenia. Del total de los 97 centros existentes en el año 2013, se seleccionaron a aquellos que hubieran ingresado 20 o más personas con sospecha de primer episodio de esquizofrenia en cualquiera de los 2 años anteriores (2011 y 2012). De esta forma, el universo de este estudio se redujo a 35 centros, y de ellos se eligieron aleatoriamente 15 centros a evaluar. Dos de estos centros presentaron dificultades que impidieron su evaluación, lo cual da una tasa de rechazo de $13,3 \%$, y en su lugar se seleccionaron aleatoriamente 2 centros de reemplazo (Tabla 1).

En cada uno de los centros seleccionados se aplicó el instrumento WHO QualityRights ${ }^{18}$. Este instrumento ha sido elaborado por la OMS y cubre 5 temas basados en la Convención de Derechos de las Personas con Discapacidad. Se evaluaron 4 temas y 18 estándares (Tablas 2 a 6). Cada estándar incluye 2 a 6 criterios y se midieron 78 criterios en total. Este instrumento se encontraba disponible solamente en idioma inglés y requirió traducción al español, evaluación de validez de contenido y pilotaje en un servicio de psiquiatría.

La aplicación del instrumento WHO QualityRights incluye observación del centro de atención en aspectos estructurales y funcionales, y entrevistas a miembros del equipo de salud, usuarios y familiares. A cada una de los entrevistados se les solicitó previamente su consentimiento informado y se tomaron todas las medidas necesarias para resguardar su identidad.

Los centros fueron evaluados durante 2 días por una dupla compuesta por un profesional de salud mental y una persona que hubiera recibido atención en un servicio de psiquiatría, los/las cuales fueron capacitados específicamente para este propósito. El evaluador profesional efectuó la observación del centro y las entrevistas a los miembros del equipo, mientras que el evaluador usuario realizó las entrevistas a los usuarios y familiares. Al final de estos procesos, ambos evaluadores definieron conjuntamente el nivel de cumplimiento (logro ausente, mínimo, parcial o total) para cada uno de los criterios de los 18 estándares estudiados. Las discordancias existentes entre los evaluadores fueron resueltas en una reunión con la investigadora responsable de la supervisión del trabajo de campo.

El estudio y los formatos de consentimientos informados fueron aprobados por el Comité de Ética de la Facultad de Medicina de la Universidad de Chile y en 7 establecimientos se requirió además la aprobación por Comités de Ética locales.

\section{Resultados}

La mediana nacional del nivel de logro con el instrumento WHO QualityRights en los servicios de Psiquiatría Ambulatoria fue de 67,8\% (siendo $100 \%$ el valor máximo de alcanzar), existiendo 
Calidad de atención y derechos de usuarios en Psiquiatría Ambulatoria - A. Minoletti et al

Tabla 1. Establecimientos evaluados y número de entrevistas efectuadas

\begin{tabular}{|c|c|c|c|c|}
\hline Establecimiento & Servicio de Salud & Usuarios & $\begin{array}{l}\text { n Entrevistas } \\
\text { Funcionarios }\end{array}$ & Familiares \\
\hline CAE ${ }^{1}$ Hospital de Quilpué & Viña del Mar-Quillota & 10 & 10 & 3 \\
\hline $\mathrm{CAE}^{1}$ Hospital Regional de Rancagua & O’Higgins & 10 & 10 & 4 \\
\hline CAE $^{1}$ Hospital Herminda Martín & Ñuble & 10 & 10 & 5 \\
\hline $\mathrm{CAE}^{1}$ Hospital Gustavo Fricke & Viña del Mar-Quillota & 11 & 10 & 5 \\
\hline $\mathrm{CDT}^{2}$ Hospital Regional de Temuco & Araucanía Sur & 8 & 9 & 3 \\
\hline $\mathrm{CAE}^{1}$ Hospital Regional de Valdivia & Valdivia & 9 & 10 & 2 \\
\hline $\mathrm{CDT}^{2}$ Hospital Grant Benavente & Concepción & 10 & 10 & 5 \\
\hline $\mathrm{CAE}^{1}$ Hospital Ancud & Chiloé & 8 & 7 & 4 \\
\hline CAE $^{1}$ Hospital San Antonio & Valparaíso San Antonio & 10 & 10 & 3 \\
\hline $\mathrm{CAE}^{1}$ Hospital Angol & Araucanía Norte & 10 & 11 & 5 \\
\hline CRS $^{3}$ Hospital Padre Hurtado & Metropolitano Suroriente & 10 & 10 & 5 \\
\hline $\mathrm{CAE}^{1}$ Instituto Psiquiátrico Dr. Horwitz & Metropolitano Norte & 10 & 10 & 5 \\
\hline $\mathrm{CDT}^{2}$ Hospital Barros Luco & Metropolitano Sur & 10 & 10 & 5 \\
\hline $\mathrm{CDT}^{2}$ Hospital Los Ángeles & Biobío & 10 & 11 & 5 \\
\hline CRS $^{3}$ Hospital El Pino & Metropolitano Sur & 10 & 10 & 5 \\
\hline Total & & 146 & 148 & 64 \\
\hline
\end{tabular}

${ }^{1}$ Centro Adosado de Especialidad; ${ }^{2}$ Centro de Diagnóstico y Tratamiento; ${ }^{3}$ Centro de Referencia de Salud.

Tabla 2. Respeto de derechos de usuarios en servicios de Psiquiatría Ambulatoria: porcentajes de logro total y por temas del instrumento WHO QualityRights

\begin{tabular}{|c|c|c|c|}
\hline Temas & Mediana & $\begin{array}{l}\text { Percentil } \\
\quad 75\end{array}$ & $\begin{array}{c}\text { Percentil } \\
25\end{array}$ \\
\hline Derecho al goce de una salud física y mental del más alto nivel posible & $73,8 \%$ & $76,8 \%$ & $70,8 \%$ \\
\hline Derecho al ejercicio de la capacidad jurídica y a la libertad y seguridad personales & $67,1 \%$ & $70,4 \%$ & $53,9 \%$ \\
\hline Protección contra la tortura y tratos crueles, inhumanos o degradantes & $78,1 \%$ & $83,3 \%$ & $67,2 \%$ \\
\hline Derecho a vivir en forma independiente y a ser incluido en la comunidad & $50,0 \%$ & $59,4 \%$ & $42,7 \%$ \\
\hline Logro total & $67,8 \%$ & $74,7 \%$ & $62,8 \%$ \\
\hline
\end{tabular}

Tabla 3. Respeto del derecho al goce de una salud física y mental del más alto nivel posible en servicios de Psiquiatría Ambulatoria: porcentajes de logro por estándares

\begin{tabular}{|c|c|c|c|}
\hline Estándares & Mediana & $\begin{array}{l}\text { Percentil } \\
75\end{array}$ & $\begin{array}{l}\text { Percentil } \\
25\end{array}$ \\
\hline $\begin{array}{l}\text { Los establecimientos están disponibles para todo aquel que requiera tratamiento } \\
\text { y apoyo }\end{array}$ & $100,0 \%$ & $100,0 \%$ & $90,6 \%$ \\
\hline $\begin{array}{l}\text { El establecimiento cuenta con personal calificado y ofrece servicios de salud mental } \\
\text { de buena calidad }\end{array}$ & $70,0 \%$ & $75,0 \%$ & $65,0 \%$ \\
\hline $\begin{array}{l}\text { Plan de "recuperación" conducido por usuario y contribuye a capacidad para vivir } \\
\text { con independencia en la comunidad }\end{array}$ & $65,0 \%$ & $72,5 \%$ & $52,5 \%$ \\
\hline La medicación psicotrópica está disponible, es asequible y se usa apropiadamente & $80,0 \%$ & $88,7 \%$ & $70,0 \%$ \\
\hline Existe disponibilidad de servicios adecuados para la salud general y reproductiva & $68,8 \%$ & $75,0 \%$ & $53,1 \%$ \\
\hline
\end{tabular}


Tabla 4. Respeto del derecho al ejercicio de la capacidad jurídica y a la libertad y seguridad personales en servicios de Psiquiatría Ambulatoria: porcentajes de logro por estándares

\begin{tabular}{lccc}
\hline Estándares & Mediana & $\begin{array}{c}\text { Percentil } \\
\mathbf{7 5}\end{array}$ & $\begin{array}{c}\text { Percentil } \\
\mathbf{2 5}\end{array}$ \\
\hline $\begin{array}{l}\text { Las preferencias de los usuarios en cuanto al lugar y forma de tratamiento son } \\
\text { siempre una prioridad }\end{array}$ & $50,0 \%$ & $62,5 \%$ & $37,5 \%$ \\
$\begin{array}{l}\text { Existen procedimientos y resguardos para evitar la detención y el tratamiento sin } \\
\text { consentimiento informado }\end{array}$ & $62,5 \%$ & $70,8 \%$ & $37,5 \%$ \\
$\begin{array}{l}\text { Los usuarios pueden ejercer su capacidad jurídica y se les da el apoyo que puedan } \\
\text { necesitar para ejercerla }\end{array}$ & $71,4 \%$ & $78,6 \%$ & $57,1 \%$ \\
$\begin{array}{l}\text { Los usuarios tienen el derecho a la confidencialidad y al acceso a su información } \\
\text { de salud personal }\end{array}$ & $62,5 \%$ & $71,9 \%$ & $56,2 \%$ \\
\hline
\end{tabular}

Tabla 5. Respeto del derecho a la protección contra la tortura y tratos crueles, inhumanos o degradantes en servicios de Psiquiatría Ambulatoria: porcentajes de logro por estándares

\begin{tabular}{|c|c|c|c|}
\hline Estándares & Mediana & $\begin{array}{l}\text { Percentil } \\
75\end{array}$ & $\begin{array}{l}\text { Percentil } \\
25\end{array}$ \\
\hline $\begin{array}{l}\text { Los usuarios tienen el derecho a estar libres de abuso verbal, mental, físico y sexual } \\
\text { y de descuido físico y emocional }\end{array}$ & $85,0 \%$ & $92,5 \%$ & $65,0 \%$ \\
\hline $\begin{array}{l}\text { Se utilizan métodos alternativos al aislamiento y contención para "atenuar el esca- } \\
\text { lamiento" de crisis }\end{array}$ & $65,0 \%$ & $80,0 \%$ & $52,5 \%$ \\
\hline $\begin{array}{l}\text { No se debe abusar de la terapia electroconvulsiva, psicocirugía y otros procedimien- } \\
\text { tos que puedan tener efectos permanentes o irreversibles, y pueden ser administra- } \\
\text { dos solamente con consentimiento informado }\end{array}$ & $100,0 \%$ & $100,0 \%$ & $81,2 \%$ \\
\hline $\begin{array}{l}\text { No se somete a ningún usuario a experimentos médicos o científicos sin su consen- } \\
\text { timiento informado }\end{array}$ & $100,0 \%$ & $100,0 \%$ & $87,5 \%$ \\
\hline $\begin{array}{l}\text { Existen medidas para prevenir la tortura o los tratos crueles, inhumanos o degra- } \\
\text { dantes y otras formas de maltrato y abuso }\end{array}$ & $50,0 \%$ & $65,6 \%$ & $41,7 \%$ \\
\hline
\end{tabular}

Tabla 6. Nivel de respeto del derecho a vivir en forma independiente y a ser incluido en la comunidad en servicios de Psiquiatría Ambulatoria: porcentajes de logro por estándares

\begin{tabular}{|c|c|c|c|}
\hline Estándares & Mediana & $\begin{array}{l}\text { Percentil } \\
\quad 75\end{array}$ & $\begin{array}{l}\text { Percentil } \\
\quad 25\end{array}$ \\
\hline $\begin{array}{l}\text { Se apoya a los usuarios para el acceso a un lugar donde vivir y contar con los recur- } \\
\text { sos financieros necesarios para vivir en la comunidad }\end{array}$ & $58,3 \%$ & $66,7 \%$ & $45,8 \%$ \\
\hline Los usuarios pueden acceder a oportunidades de educación y empleo & $50,0 \%$ & $62,5 \%$ & $29,2 \%$ \\
\hline $\begin{array}{l}\text { Se apoya el derecho de los usuarios a participar en la vida política y pública y en el } \\
\text { ejercicio de la libertad de asociación }\end{array}$ & $41,7 \%$ & $52,1 \%$ & $33,3 \%$ \\
\hline $\begin{array}{l}\text { Se apoya a los usuarios en la participación en actividades sociales, culturales, reli- } \\
\text { giosas y de recreación }\end{array}$ & $50,0 \%$ & $56,2 \%$ & $41,7 \%$ \\
\hline
\end{tabular}

variaciones en los puntajes obtenidos por los distintos centros evaluados, con un percentil 25 (P25) de $62,8 \%$ y un percentil 75 (P75) de $74,7 \%$ (Tabla 2). Existieron diferencias también en el nivel de logros entre los 4 temas que incluye el instrumento
(Tabla 2), alcanzando los valores más altos los temas sobre el derecho al goce de una salud del nivel más alto posible, con una mediana nacional de $73,8 \%$, y el derecho a la protección de tratos crueles, degradantes, violentos y abusivos, con una 
mediana de 78,1\%. El derecho a vivir en forma independiente y a ser incluido en la comunidad es el que tuvo el puntaje más bajo de los cuatro temas, con una mediana de 50\% (Tabla 2), y es el que además presentó la mayor variación de nivel de logros entre los centros, con $35,4 \%$ el de menor nivel y $66,7 \%$ el de mayor nivel.

Las medianas nacionales de cumplimiento de los estándares para el derecho al goce de una salud del nivel más alto posible fluctuaron entre $65 \%$ y $100 \%$, siendo el estándar "plan de recuperación conducido por el usuario" el de menor logro (Tabla 3). El criterio de este estándar con puntaje más bajo fue "participación activa del usuario en su plan de recuperación". El otro estándar de este tema que mostró un puntaje bajo (mediana de $68,8 \%$ ), fue la "disponibilidad de atención para salud general"; el criterio con el puntaje más bajo para este estándar fue el que se refiere a la educación y promoción de la salud.

El estándar del "derecho a la capacidad jurídica y a la libertad y seguridad personal" con menor nivel de logro fue "prioridad de las preferencias del usuario para lugar y forma del tratamiento", con una mediana de 50\% (Tabla 4). Los otros dos estándares con puntaje bajo fueron "resguardos para evitar la detención y el tratamiento sin consentimiento" (mediana de 62,5\%) y "confidencialidad y acceso a la ficha clínica" (mediana de 62,5\%).

En relación al derecho a la protección de tratos crueles, degradantes, violentos y abusivos (Tabla 5), el estándar más bajo fue "medidas para prevenir tortura o tratos crueles o inhumanos y otras formas de maltrato" (mediana de 50\%). Los criterios con puntaje más bajo para este estándar fueron "acceso de los usuarios a representantes legales y a defensores", y "supervisión del establecimiento por una autoridad independiente al sistema de salud para prevenir maltratos". El segundo estándar con puntaje más bajo fue "uso de métodos alternativos al aislamiento y la contención" (mediana de 65\%).

La Tabla 6 muestra que los cuatro estándares del derecho a vivir en forma independiente y a ser incluido en la comunidad presentaron una mediana de logro nacional bajo $60 \%$, mientras que sólo 2 de los 18 estándares de los otros temas se encontraban bajo este porcentaje (Tablas 3, 4 y 5). Estos 4 estándares también presentaron una variación alta de cumplimiento entre los distintos centros. El estándar con la menor mediana fue "apoyo que se entrega a los usuarios para participar en la vida política y pública y en la libertad de asociación". Los criterios con puntaje más bajo para este estándar se refieren a la entrega de información y de apoyos para ejercer el derecho a voto y participar en organizaciones.

\section{Discusión}

La información que entrega este estudio permite caracterizar algunos de los logros y debilidades que ha alcanzado Chile en los servicios de Psiquiatría Ambulatoria del sistema público. Entre los logros, se destacan el relativamente alto puntaje que alcanzan estos servicios en los temas de derecho al goce de una salud del más alto nivel posible y de protección contra la tortura y tratos crueles, inhumanos o degradantes. En el primer tema, el buen desempeño tiene que ver principalmente con el nivel de cumplimiento de estándares relacionados con la disponibilidad para atender sin discriminaciones y con la disponibilidad de medicación psicotrópica, mientras que en el segundo tema se relaciona con el uso habitual del consentimiento informado para procedimientos de riesgo (terapia electroconvulsiva, psicocirugía, etc.) y en investigación, así como el trato adecuado hacia los usuarios (sin abusos ni descuidos mayores).

A su vez, las debilidades se concentran mayoritariamente en el tema del derecho a vivir en forma independiente y a ser incluido en la comunidad, donde la mediana de logro es de solamente 50\% y la cuarta parte de los centros alcanza un logro de $42,7 \%$ o menos. Este bajo desempeño se relaciona con la práctica habitual de los servicios de psiquiatría de entregar prestaciones "clínicas", biomédicas y psicológicas, en desmedro de las prestaciones "comunitarias". Son precisamente estas últimas las que contribuyen a apoyar a las personas con enfermedades mentales severas en sus necesidades de vida diaria en la comunidad, tales como tener un lugar donde vivir, acceder a educación y empleo, y participar en diferentes actividades.

Además, dos indicadores correspondientes a otros temas requieren una consideración especial por haber presentado un bajo nivel de logro (50\%). Uno de ellos, "las preferencias de los usuarios en cuanto al lugar y forma de tratamiento son siempre una prioridad", se relaciona fundamen- 
talmente con las percepciones y actitudes que los profesionales de salud tienen hacia las personas con enfermedades o discapacidades mentales, mientras que el otro, "existen medidas para prevenir la tortura o los tratos crueles, inhumanos o degradantes y otras formas de maltrato y abuso", tiene que ver más bien con la forma que los sistemas de salud y justicia implementan mecanismos para evitar este tipo de tratos.

No se encontró en la literatura otros estudios de aplicación del instrumento WHO QualityRights en servicios de Psiquiatría Ambulatorios que permitiera comparar nuestros resultados con el nivel de respeto de derechos de usuarios en otros países. En comparación con las evaluaciones de servicios de hospitalización psiquiátrica realizadas con este instrumento, nuestros resultados son similares a los encontrados en Andalucía ${ }^{19}$ y superiores a los de los hospitales de Serres en Gre$\mathrm{cia}^{21}$ y Hargeisa Group en Somalilandia ${ }^{20}$. Se debe tener en cuenta, sin embargo, que generalmente el nivel de respeto de derechos de usuarios es menor en servicios psiquiátricos de hospitalización que en los ambulatorios ${ }^{12,22}$. En países de Europa Occidental se han realizado varios estudios de servicios ambulatorios de Psiquiatría y de otras especialidades, utilizando métodos diferentes al QualityRights, en los que se han medido el respeto de algunos de los derechos evaluados en nuestra investigación, tales como consentimiento informado, trato adecuado a los usuarios, consideración de las preferencias de los usuarios, apoyo para la inclusión social, acceso a la ficha clínica y confidencialidad $^{22-29}$. En estos estudios se aprecia que los procedimientos desarrollados y el nivel de resguardo y ejercicio de derechos son superiores a los resultados encontrados en los servicios de Psiquiatría Ambulatoria chilenos.

La principal debilidad de este estudio se refiere a la muestra utilizada, la cual es sólo representativa de los establecimientos del país con servicios de Psiquiatría que atienden una alta cantidad de usuarios. Queda la interrogante para futuros estudios si el nivel de cumplimiento de los estándares en estos centros es similar o diferente al que se pueda encontrar en los centros de salud mental comunitarios o consultorios de hospitales generales de ciudades más pequeñas, los cuales tienen un territorio y una población más acotados y donde generalmente realizan un mayor número de intervenciones comunitarias.
Los hallazgos de este estudio pueden contribuir a la formulación de recomendaciones con el fin de mejorar el desempeño de los servicios de Psiquiatría a nivel de los países con mayor desarrollo en esta área y así contribuir a una recuperación integral de las personas con enfermedades mentales, así, por ejemplo:

1. A nivel de legislación: Se podrían realizar modificaciones que garanticen los derechos de acuerdo a la Convención de Derechos de las Personas con Discapacidad y los estándares de la OMS definidos en el instrumento QualityRights.

2. A nivel de politicas nacionales: Es necesario y factible el desarrollo de una estrategia de mejora continua de calidad y respeto de derechos de usuarios en los centros de salud mental, en la cual se adopten los estándares que ha propuesto la OMS.

3. A nivel de programas de salud mental locales: Es posible reforzar el trabajo comunitario de los equipos de salud mental, generando procesos de aprendizaje y buenas prácticas en el apoyo a las personas en sus procesos de inclusión en la comunidad y ejercicio de derechos humanos.

4. A nivel de los servicios de Psiquiatría: Se pueden implementar una serie de medidas específicas para el mejoramiento de la calidad y respeto de derechos, tales como:

- Capacitación teórico-práctica de los equipos de atención sobre los estándares incluidos en el WHO QualityRights.

- Autoevaluación y evaluación externa con el Instrumento QualityRights.

- Formulación e implementación de planes de mejora de cumplimiento de estándares.

- Incorporación de "facilitadores de derechos de los usuarios", los cuales deben ser independientes del equipo de atención (ej. usuarios con un alto nivel de recuperación, voluntarios de la comunidad, etc.).

5. A nivel de usuarios de servicios de salud mental: Se pueden beneficiar de una mayor información de sus derechos en la atención de salud mental y de cómo ejercerlos al interior de los servicios de Psiquiatría. Algunas alternativas para este efecto son:

- Folletos, afiches y vídeos para sala de espera.

- Talleres de formación en el ejercicio de derechos. 
- Asambleas de usuarios para el análisis del funcionamiento del servicio.

6. A nivel de investigación: Nuevos estudios sobre respeto de derechos en diferentes establecimientos de salud mental (ej. centros de salud mental comunitarios, unidades de psiquiatría de corta estadía en hospitales generales, etc.) $\mathrm{y}$ de intervenciones para mejorar este respeto podrían generar conocimientos valiosos para mejorar la atención. Además, es interesante desarrollar en mayor profundidad la línea metodológica iniciada en este estudio de incorporar a usuarios en las investigaciones sobre el funcionamiento de servicios de salud.

\section{Referencias}

1. Kilbourne AM, Keyser D, Pincus HA. Challenges and opportunities in measuring the quality of mental health care. Can J Psychiatry 2010; 55 (9): 549-57.

2. Legido-Quigley H, McKee M, Nolte E, Glinos IA. Assuring the quality of health care in the European Union: A case for action. Copenhagen: WHO Regional Office for Europe, on behalf of the European Observatory on Health Systems and Policies; 2008.

3. Ganju V. Mental health quality and accountability: The role of evidence-based practices and performance measurement. Adm Policy Ment Health \& Ment Health Serv Res 2006; 33: 659-65.

4. Wobrock T, Weinmann S, Falkai P, Gaebel W. Quality assurance in psychiatry: quality indicators and guideline implementation. Eur Arch Psychiatry Clin Neurosci 2009; 259 (Suppl 2): S219-S26.

5. Raleigh VS, Foot C. Getting the measure of quality: Opportunities and challenges. London: The King's Fund; 2010.

6. Araya R, Alvarado R, Minoletti A. Chile: An ongoing mental health revolution. The Lancet 2009; 374 (22): 597-8.

7. Minoletti A, Sepúlveda R, Horvitz-Lennon M. Twenty years of mental health policies in Chile: Lessons and challenges. Int J Men Health 2012; 41 (1): 21-37.

8. Minoletti A, Rojas G, Horvitz-Lennon M. Integrating mental health into primary care: Lessons from the experience of Chile. Sorel E, editor. 21st Century Global Mental Health. Burlington (Ma): Jones \& Bartlett Learning; 2012. p. 255-81.

9. Minoletti A, Alvarado R, Rayo X, Minoletti M. Evaluación de sistemas de salud mental en Chile: Segundo informe. Santiago, Chile: Ministerio de Salud; 2014.
10. Alvarado R, Minoletti A, Aliste F, Sepúlveda R, Madariaga C, Caro J. Evaluación de las redes de atención para personas con primer episodio de esquizofrenia en Chile: Informe general (Proyecto FONIS SA08I20033). Santiago, Chile: Escuela de Salud Pública Dr. Salvador Allende, Universidad de Chile; 2010.

11. Killaspy H, White S, Wright C, Taylor T, Turton P, Schützwohl M, et al. The development of the Quality Indicator for Rehabilitative Care (QuIRC): a measure of best practice for facilities for people with longer term mental health problems. BMC Psychiatry 2011; 11: 35. Disponible en: http://www.biomedcentral.com/1471244X/11/35 [Consultado el 26 de diciembre de 2014].

12. Pincus HA, Spaeth-Rublee B, Watkins KE. The case for measuring quality in mental health and substance abuse care. Health Affairs 2011; 30 (4): 730-6.

13. Spaeth-Rublee B, Pincus HA, Huynh PT. Measuring quality of mental health care: a review of initiatives and programs in selected countries. Can J Psychiatry 2010; 55 (9): 539-48.

14. Watkins K, Horvitz-Lennon M, Caldarone LB, Shugarman LR, Smith B, Mannle TE, et al. Developing medical record-based performance indicators to measure the quality of mental healthcare. J Healthc Qual 2010; 33 (1): 49-67.

15. Gostin LO, Gable L. The human rights of persons with mental disabilities: A global perspective on the application of human rights principles to mental health. Washington DC: Georgetown University Law Center; 2004. Disponible en: http://scholarship.law.georgetown. edu/facpub/98 [Consultado el 26 de diciembre de 2014].

16. Drew N, Funk M, Tang S, Lamichhane J, Chávez, E, Katontoka S, et al. Human rights violations of people with mental and psychosocial disabilities: an unresolved global crisis. The Lancet 2011; 378 (9803): 1664-75.

17. Organización de las Naciones Unidas. Convención de las Naciones Unidas sobre los derechos de las personas con discapacidad y su protocolo facultativo. Santiago, Chile: Ministerio de Relaciones Exteriores; 2008. Disponible en: http://www.leychile.cl/Navegar/?idNor$\mathrm{ma}=278018$ \&idVersion=2008-09-17\&idParte [Consultado el 26 de diciembre de 2014].

18. World Health Organization. WHO QualityRights tool kit: assessing and improving quality and human rights in mental health and social care facilities. Geneva: World Health Organization; 2012. Disponible en: http://www. who.int/mental_health/publications/QualityRights_ toolkit/en/ [Consultado el 26 de diciembre de 2014].

19. Gobierno del Principado de Asturias. Evaluación de los servicios de salud mental del Principado de Asturias: Evaluación de derechos humanos y calidad en 
instalaciones con internamiento. Oviedo, Asturias: Gobierno del Principado de Asturias; 2010. Disponible en: http://www.asturias.es/portal/site/astursalud/menuitem.2d7ff2df00b62567dbdfb51020688a0c/?vgnextoid=51ee169cb4ff0310VgnVCM10000098030a0aRCRD [Consultado el 26 de diciembre de 2014].

20. Currie J. Evaluación de calidad y derechos humanos en el Servicio de Psiquiatría del Hospital Hargeisa Group en Somalilandia. Comunicación personal. 2012.

21. Nomidou A. Standards in mental health facilities-an in depth case study in Greece using the WHO QualityRights tool. J Public Ment Health 2013; 12 (Iss: 4): 201-11.

22. Bramesfeld A, Wedegärtner F, Elgeti H, Bisson S. How does mental health care perform in respect to service users' expectations? Evaluating inpatient and outpatient care in Germany with the WHO responsiveness concept. BMC Health Services Research 2007; 7: 99-110.

23. Roy A, Jain S, Roy A, Ward F, Richings C, Martin M, Roy M. Improving recording of capacity to consent and explanation of medication side effects in a psychiatric service for people with learning disability: Audit findings. J Intellect Disabil 2011; 15 (2): 85-92.

24. Johnstone C, McCartney G. A Patient Survey Assessing the Awareness and Acceptability of the Emergency Care
Summary and Its Consent Model in Scotland. Perspect Health Inf Manag 2010; Spring: 1-7.

25. Eldh AC, Ehnfors M, Ekman I. The phenomena of participation and non-participation in health care-experiences of patients attending a nurse-led clinic for chronic heart failure. Eur J Cardiovasc Nurs 2004; 3 (3): 239-46.

26. Eldh AC, Ehnfors M, Ekman I. The meaning of patient participation for patients and nurses at a nurse-led clinic for chronic heart failure. Eur J Cardiovasc Nurs 2006; 5 (1): 45-53.

27. Woodrow SR, Jenkins AP. How thorough is the process of informed consent prior to outpatient gastroscopy? A study of practice in a United Kingdom District Hospital. Digestion 2006; 73 (2-3): 189-97.

28. Mold F, Ellis B, de Lusignan S, Sheikh A, Wyatt JC, Cavill $\mathrm{M}$, et al. The provision and impact of online patient access to their electronic health records (EHR) and transactional services on the quality and safety of health care: systematic review protocol. Inform Prim Care 2012; 20 (4): 271-82.

29. McKinstry B, Watson P, Pinnock H, Heaney D, Sheikh A. Confidentiality and the telephone in family practice: a qualitative study of the views of patients, clinicians and administrative staff. Fam Pract 2009; 26 (5): 344-50. 